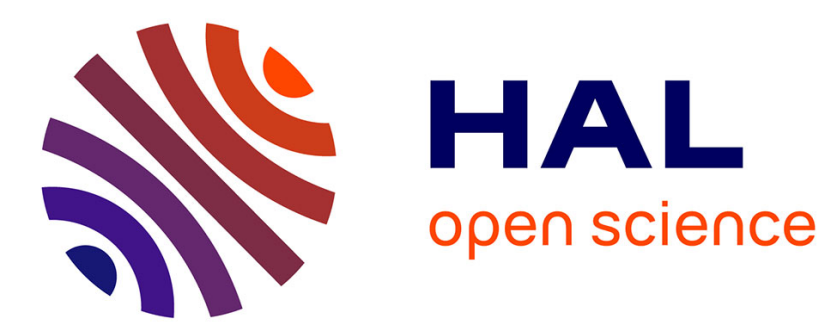

\title{
Structural diagnosability analysis. Application to an induction motor
}

\author{
Naouel Kaci, B. Ould Bouamama, I Boussaada, Achour Debiane
}

\section{To cite this version:}

Naouel Kaci, B. Ould Bouamama, I Boussaada, Achour Debiane. Structural diagnosability analysis. Application to an induction motor . 2017 IEEE 11th International Symposium on Diagnostics for Electrical Machines, Power Electronics and Drives (SDEMPED), Aug 2017, Tinos, Greece. hal$01658216 \mathrm{v} 2$

\section{HAL Id: hal-01658216 https://hal.science/hal-01658216v2}

Submitted on 14 Jan 2018

HAL is a multi-disciplinary open access archive for the deposit and dissemination of scientific research documents, whether they are published or not. The documents may come from teaching and research institutions in France or abroad, or from public or private research centers.
L'archive ouverte pluridisciplinaire HAL, est destinée au dépôt et à la diffusion de documents scientifiques de niveau recherche, publiés ou non, émanant des établissements d'enseignement et de recherche français ou étrangers, des laboratoires publics ou privés. 


\section{Structural diagnosability analysis. Application to an induction motor}

\author{
N. Kaci \\ IPSA, Bis, 63 Boulevard de Brandebourg, \\ 94200 Ivry-sur-Seine Email: naouel.kaci@ipsa.fr
}

\author{
B. Ould Bouamama \\ Univ. Lille, UMR 9189-CRIStAL Laboratory \\ F-59000 Lille, France. Email: belkacem.ouldbouamama@univ-lille1.fr
}

\author{
I. Boussaada \\ IPSA 63 Boulevard de Brandebourg, \\ 94200 Ivry-sur-Seine Email: islam.boussaada@ipsa.fr \\ A. Debiane \\ CERTIA Enterprise for research in industrial technology \\ Rue des Artisans, 93160 Noisy-le-Grand. Email: adebiane@certia.fr
}

\begin{abstract}
The presented research is carried out within the framework of a global industrial project "Electro-Mechanical Actuator Health Management" with french company CERTIA. The objective is the realization of an auto-diagnosis embedded module for health monitoring and reconfiguration of ElectroMechanical Actuator (EMA) including two operating modes: Test checking before taking off (evaluation and health management, i.e evaluation of the rate of degradation) and online supervision including (i)fault detection and isolation (to avoid the irreversible jamming default for instance) and (ii) fault tolerant control and/or reconfiguration in faulty situation (how to continue to control the system en degraded mode ?). The present paper concerns the first part of the project. The innovative interest concerns use of bond graph model as unified and multidisciplinary tool not only for modeling but also for structural diagnosability analysis (which faults which may affect component including sensors can be detected an isolated ?) and sensor placement proposition to make the system diagnosable Without any need of numerical calculation. An application to an induction motor as main component in an EMA is used for illustration.
\end{abstract}

\section{INTRODUCTION AND GENERAL ISSUES}

The complexity and safety critical needs of systems such as automotive systems or aircraft systems have motivated the need for automated monitoring and diagnosis as part of the intelligent control loop to insure the dependability and availability of components in faulty and healthy situation. The need for safety and efficient control under a variety of operating conditions requires online Fault Detection and Isolation (FDI) procedures that can inform intelligent Fault Tolerant and Fault Adaptive Control (FTC and FAC) schemes. The FDI methodology is based on the comparison between the actual system behavior (given by a sensors) with the normal operation behavior (provided by a model). When the observed behavior differs from the nominal behavior, the diagnosis method uses this difference, expressed as a non-zero residual vector (named alarm) as the basis for the isolation step to identify which component is faulty using a logic procedure. Depending on the kind of used models, two types of methods have been developed : model-based approaches (using analytical models) [1] and knowledge-based (using signal or pattern recognition) [2], named non-model-based approaches. In contrary to the model-based approaches where a prior, knowledge about the model of the system is assumed, in non-model-based methods only the availability of large amount of historical process data is assumed. However, the isolation performances of no model based methods need historical data in normal and in abnormal situations, thus every fault mode has to be represented (which is not always possible in the real systems). This is why FDI model based approaches is an interesting alternative.

A key factor determining the performance of analytical FDI techniques (observers, parity space...) is the model accuracy but need numerical values of model parameters for final evaluation. However, graphical methods present a big interest because their models capture only system structure by representing the system variables and set of behavior equations just as nodes and arc. Furthermore, the graph structure is independent of the numerical values of the system parameters. This is why graphical methods are well suited for model based structural diagnosis. In this context among qualitative models (digraph, bipartite graph, and signed digraph) bond graph as a powerful and multidisciplinary tool for modeling is well suited because of its causal, behavioral and structural properties. A comparison of this tool with others graphical models for FDI is given in [3].

The presented research is carried out within the framework of a global industrial project "Electro-Mechanical Actuator Health Management (EMAHM)" with french company CERTIA specialized in avionic test bench. The objective is the realization of an auto-diagnosis embedded module for health monitoring and reconfiguration of Electro-Mechanical Actuator (EMA) including two operating modes: Test checking before taking off (evaluation and health management, i.e evaluation of the rate of degradation) of the EMA and online supervision including fault detection and isolation (to avoid the irreversible jamming default for instance) and fault tolerant control and/or reconfiguration in faulty situation (how to continue to control the system en degraded mode ?).

Fig.1 illustrates a different stages for the realization of this project. This scheme is essentially composed of two parts. The first part realized offline, concerns diagnosability analysis (i.e., the ability to determine which component can be monitored without any need of numerical calculations, and 
before implementation).

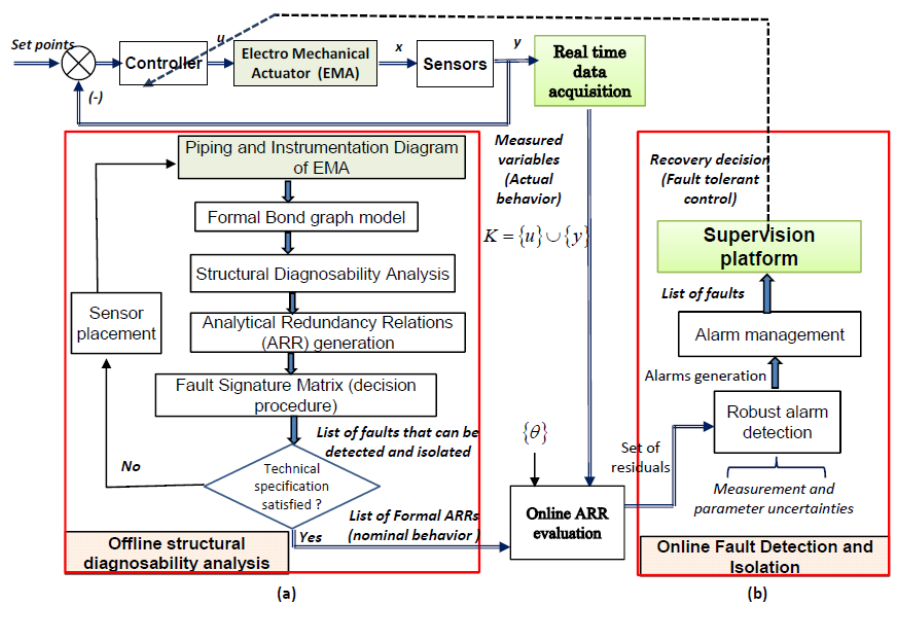

Fig. 1. Offline (a) and online (b) design steps of EMA supervision

Comparing with analytical approaches, the modular and functional aspect of bond graphs, allows for direct mappings between generated fault indicators (ARR) and specific sensor, actuator and component faults in the system [4]. While the detectability and isolability performances based on Fault Signature Matrix, performed offline and directly from the graphical model because of its causal properties. At the end, sensor placement can be proposed graphically in the model to make the system diagnosable according to the fixed technical specifications. Finally the generated set of formal ARRs are evaluated (if parameter values are available) to produce numerical residuals for online supervision including FDI and recovery decisions.

The present paper concerns the first part of the project, and deals with use of bond graph model as unified and multidisciplinary tool not only for modeling but also for structural diagnosability analysis (which faults which may affect component including sensors can be detected an isolated ?) and sensor placement proposition to make the system diagnosable. An application to an induction motor as main component in an EMA is used for illustration. The paper is organized as follows. The second section deals with Bond Graph (BG) theory and structural analysis basis. In the third part, the diagnosability performances based on BG is developed. The methodology is applied to an induction motor as main device for driving an EMA. The fourth section present conclusion and future work.

\section{BOND GRAPH STRUCTURAL ANALYSIS BASIS}

\section{A. Bond Graph theory}

Definition 1: The key of bond graph modeling invented by Paynter [5] is the representation (by a bond) of exchanged power as the product of two generic power variables (named efforts $e$ and flows $f$ ) with elements acting between these variables and junction structures to reproduce the global system as interconnected subsystems.

Comparing with other graphical models (SDG [6], digraphs, bipartite graphs, [7]) where vertices are variables and the edges represent mutual influence between those variables, BG is also a graph $G(V, E)$, but the vertices $V$ are BG elements corresponding to physical components, subsystems and connection elements, and where $E$ (Edges) is a set of oriented bonds used to represent power exchanges between vertices. In BG representation, nodes are elementary elements represented by the set $V=\{R \cup C \cup I \cup T F \cup G Y \cup S e \cup S f \cup D e \cup D f \cup J\}$. The $R$ - element represents a passive energy dissipation phenomena (electrical resistor, mechanical friction), while $C$, and $I$ elements model the passive energy storage under potential (electrical capacity, tank, spring) and kinetic (inductance, mechanical inertia) respectively. $T F$, and $G Y$ represent transformers and gyrators respectively used to model energy transformation from one form into another. The system inputs are modeled using simple effort source $(S e)$ and flow source $(S f)$ or controlled (modulated) sources $(M S e)$ and $M S f$ ) elements. Effort sources can be current generator or applied velocity and flow sources are for example generator of voltage, gravity force, pressure pump. Junction 1 (resp, 0 ) is used to regroup BG elements which share the same flow (resp, the same effort). Sensors are modeled by detectors of effort $(D e)$ and flow $(D f)$ they are connected to the junction 0 (resp, 1) by an arrow to show that they exchange information (not a power).

The exchanged power between two systems $V_{1}$ and $V_{2}$ (Fig. 2(a)) is the product of two conjugated power variables: a potential variable (e.g.pressure, electrical potential, temperature, chemical potential, force, etc.) called effort $(e)$ placed above the bond and a derivative of extensive variable (e.g. volume flow, current, entropy flow, velocity, molar flow, etc.) referred to as flow $(f)$, placed below the bond. The direction of the positive power is indicated by the half arrow. Independently of this power direction the algorithmic level of modeling is represented by the causality concept shown by the causal stroke place.

\section{- Causality concept}

The physics is naturally acausal. But, if we need to simulate the physical phenomena (the model); we have to decide in which order the variables (effort and flow) will be computed. Consequently we need to make a series of cause and effect decisions: this is a concept of causality. The causality is denoted by a cross-stroke. By convention, this causal cross is placed near (respectively far) the element for which the effort (respectively flow) is known. This leads to a bloc diagram simulation (Fig. $2 b_{1}$ ). As illustrated by the electrical resistance $\mathbf{R}$ supplied by a battery $\mathbf{S}$ (Fig. 2(a $\left.a_{1}\right)$ ) where effort $e$ and flow $f$ power variables represent voltage and current. The bond graph can be acausal ( $e-R_{1} . f=0$ ), or causal (Fig. $\left.2\left(b_{1}\right)\right)$, with two possible causal equations: $f=\frac{e}{R_{1}}$ if $\mathrm{S}$ is a voltage source or $e=R_{1} . f$ if $\mathrm{S}$ is a current source. This concept of causal properties constitutes an important structural and causal characteristics allowing to directly determine from the graphical representation observability, controllability and monitorability structural properties. Notion of a causal path is given while it will be used in the procedure for manual 
derivation of equations from bond graphs or unknown variables elimination in diagnosis bond graph model based. Note that causally completed bond graphs may be converted into a block diagram if needed. Indeed, the determination of causes and effects (by a covering causal path) in the system is directly deduced from the graphical representation.

Definition 2: (Causal path). A sequence of bonds from one power port of an element to a power port of another element is called a causal path if there is no 2-port gyrator in between and if all bonds have their causal stroke at the same end. If the bonds of a causal path connect only elements of the junction structure and if the causal path is closed, then it is called a causal loop.

In Fig. $2 a_{2}, b_{2}$ ) are shown the causal paths (represented by dashed arrows) for the given bond graphs.

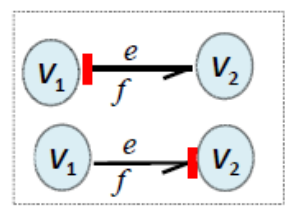

(a)

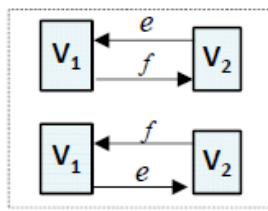

(b)

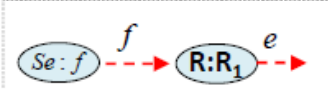

$\left(a_{2}\right)$

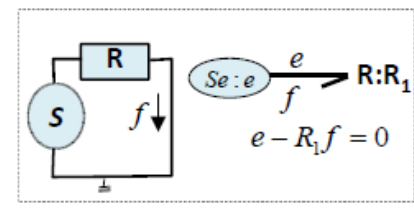

$\left(a_{1}\right)$

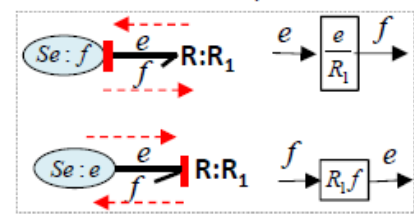

$\left(\mathrm{b}_{1}\right)$

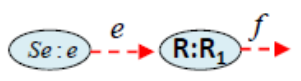

$\left(b_{2}\right)$
Fig. 2. Causal BG model (a) and corresponding bloc diagrams (b) illustrated by an electrical example (a1) and (b1) and their causal paths (a2, b2)

\section{B. Structural model}

The structural behavior model of any physical system can be defined by a pair $(C, Z)$, where $\left\{Z=z_{1}, z_{2}, \ldots, z_{n}\right\}$ is a set of variables and parameters, and $\left\{C=c_{1}, c_{2}, \ldots, c_{n m}\right\}$ is a set of constraints. The constraints may be expressed in several forms like algebraic, difference equations, rules, etc. This structural model can be represented by an adjacency boolean matrix $M$ (incidence matrix), whose elements $m_{i j}$ are defined as follows:

$$
m_{i j}=\left\{\begin{array}{l}
\left.1 \text { if } \mathrm{z}_{\mathrm{i}} \in c_{j}\right) \\
0 \text { else }
\end{array}\right.
$$

This matrix is used in diagnosis to find the redundancies based on bipartite graph realized from analytical model. In the present research, all redundancies are deduced directly from the bond graph model without any need of analytical calculation. The variables which can be quantitative, qualitative, fuzzy,... consist of two subsets :

$$
Z=\{X\} \cup\{K\}
$$

where $\{X\}$ are unknown variables and $\{K\}$ are known variables which consists of control variables $U$ and subset of measured variables $Y$. The set of constraints, $C$, from a bond graph theory include information about the structure $C_{s}$, the behavior $C_{b}$, the measurement $C_{m}$ and the control system $C_{c}$ :

$$
C=\left\{C_{s}\right\} \cup\left\{C_{b}\right\} \cup\left\{C_{m}\right\} \cup\left\{C_{c}\right\}
$$

Structural equations $\left\{C_{s}\right\}$ represent a set of conservation laws and are deduced from the junction equations, transformers and gyrators. The set $\left\{C_{b}\right\}$ is associated with the behavior model (expressing how the energy is transformed). In a BG model, they describe the physical phenomena which are represented by $\mathrm{BG}$ elements ( $\mathbf{R}, \mathbf{I}$ and $\mathbf{C})$. Measurement model $\left\{C_{m}\right\}$ expresses the way in which the sensors transform some state variables of the process into output signals which can be used for FDI and control purposes. In BG models, the sensors are represented as detectors of flow $(D f)$ and detectors of effort $(D e)$. The unknown variables $\{X\}$ are the pair of power variables that label the bonds: $X=\left\{e_{i}, f_{i}\right\}, i=1 \ldots n$, where $n$ is the number of passive BG elements ( $\mathbf{R}, \mathbf{I}$ and $\mathbf{C})$.

The input variables are represented by the flow $(S f)$ and the effort $(S e)$ sources and modulated flow $(M S f)$ and effort ( $M S e$ if they are controlled. Finally the set of known variables $\{K\}$ are:

$$
K=\left\{D_{e}\right\} \cup\left\{D_{e}\right\} \cup\{S f\}\{S e\}\{M S f\}\{M S e\}
$$

\section{Canonical decomposition}

Recall first that any subgraph $G(C x, X, A)$ where $C x, X$ and $A$ are subset of constraints matched by unknown variables, unknown variables and edges, is a matching on $G(C x, X, A x)$ if and only if : (i) $A \subset A x$, (ii) $\forall a 1, a 2 \in A a 1 \neq a 2$, (iv) $C x(a 1) \neq C x(a 2)$ and $X(a 1) \neq X(a 2)$ where $X(a)$ and $C x(a)$ are respectively starting and ending nodes. In fact, a matching is a set of pairs constraint, variables $\left(c_{i}, x_{i}\right)$ so that the variable $x_{i}$ can be computed by solving the constraint $c_{i}$, under the hypothesis that all other variables are known.

In [7], it is shown that any system to be monitored can be decomposed into three main parts: (i) The structurally overconstrained subsystem $M^{+}$, where the matching is complete with respect to unknown variables $X$ but incomplete with respect to the constraints $C$. Otherwise, a set $C$ of equations is structurally over determined if $C$ has more equations than unknowns variable $X$. (ii) The structurally just-constrained subsystem $M^{0}$, where the matching is complete with respect to variables $X$ and to the constraints $C$, this means that the number of equations in the system is equal to the number of variables. (iii) The structurally under-constrained $\mathrm{M}^{-}$, where the matching is complete on the constraints $C$ but not on the variables $X$ : the number of variables is greater than the number of equations. The under-constrained subsystem is not observable, and not monitorable. Recall that observability is a necessary but not sufficient of diagnosability condition :

- Under derivative causality, the system is structurally observable if and only if : (i) All the unknown variables are 
reachable from the known ones (measure), (ii) the over constrained and just-constrained subsystems are causal (no differential loop, all variables can be matched using causal matching), (iii) the under-constrained subsystems is empty (all variables can be matched.

\section{Structural diagnosability}

The conditions for a fault $\varphi$ to be monitorable are : (i) The subsystem is observable. (ii) The fault $\varphi$ belongs to the structurally observable over constrained part of the subsystem to be monitored. A method based graph theory loses sometimes certain information when writing the structure of a graph representing system structure. This is due to the fact that construction is generally from state equation, which does not explain all relations constitutive of the system studied. Furthermore, Dulmage-Mendelsohn decomposition based on structural model needs determination of the whole of analytical equations (constraints) and corresponding variables: this task is not trivial for complex systems. In the next subsection, it will be shown how the $\mathrm{BG}$ representation can be used in order to deduce different subsystems directly from BG model.

\section{E. Fault indicators generation from bond graph}

In [8], the BG model has been used for ARR generation for determinist FDI. Classically, an ARR is a constraint derived from an over-constrained subsystem and expressed in terms of known variables of the process. In a BG sense, an $A R R=f(S S e, S S f, S e, S f, M S e, M S f, \theta)$, where $\theta$ is the parameters vector, $S S e$ (SSf) are signal sources of effort (flow) that are obtained from the sensors of effort $D e$ (flow $D f$ ). $S e$, and $M S e$ ( $S f$, and $M S f)$ symbolize the system inputs of effort (flow). The causal properties of a BG model can be exploited for generation of ARRs directly from the model in derivative causality. For this task, dualizing an effort (or flow) detectors transforms it into a signal source (SSe, SSf). This imposed signal is the starting point for the elimination of unknown variables using covering causal paths from unknown variables to known ones (sensors and input sources) contained in the structural constraints of junctions "0" and "1". The algorithm of ARRs generation can be found in [8]. The procedure is implemented in a software developed by one of the authors as a toolbox [9]. On a bipartite graph and incidence matrices, the starting point for the elimination of unknown variables is difficult to find because there is many possibilities of matching. The determination of an over-constrained matching for ARRs generation using bond graph is done by the elimination of unknown variables containing in the structural constraints issued from "0" and " 1 " junctions.

\section{F. Diagnosability performances}

- Structural detectability from bond graph model

Proposition 1: A fault $F$ which may affect a component $C O M P_{i}$ belonging to a subsystem modeled by a bond graph model in derivative causality is detectable if and only if there is at least one sensor (detector of effort or flow : $\left|C_{m}\right| \geq 1$.
Proof 1 Consider now the global bond graph model of the system to be monitored which consists of $\ell$ junctions. The cardinal of the unknown variables $|X|$ and the cardinal of constraints can be given through the following relations:

$$
\begin{aligned}
& \operatorname{card}(C)=|C|=\sum_{j=1}^{\ell}\left|C^{j}\right|=\ell+\sum_{i=1}^{\ell}\left(\left|C_{b}^{j}\right|+\left|C_{m}^{j}\right|\right) \\
& \operatorname{card}(X)=|X|=\sum_{j=1}^{\ell}\left|X^{j}\right|=\ell+\sum_{i=1}^{\ell}\left(\left|C_{b}^{j}\right|\right)
\end{aligned}
$$

Then, if is added set of sensors of cardinal $\operatorname{card}\left(C_{m}\right)$, then the cardinality of constraints will be

$$
\operatorname{card}(C)=|C|=|X|+|C|=\operatorname{card}(X)+\operatorname{card}\left(C_{m}\right) .
$$

While the condition of over determination is $\operatorname{card}\left(C_{m}\right) \neq 0$, this means that there is at least one sensor.

- Structural isolability

The equations of power balance on the junctions constitute the candidate ARRs [9]. Consider m candidates ARRs. During the covering causal paths for ARR generation (which leads to an oriented graph where nodes are constraints represented by bond graph elements), a set of components $\left\{E_{i j}\right\}$ (noted $C O M P(A R R))$ will be covered during this sequence:

$$
\left\{\begin{array}{l}
\operatorname{COMP}\left(A R R_{1}\right)=\left\{\begin{array}{lllll}
E_{11} & \ldots & E_{1 j} & \ldots & E_{1 n_{1}}
\end{array}\right\} \\
\ldots \\
\operatorname{COMP}\left(A R R_{m}\right)=\left\{\begin{array}{lllll}
E_{m 1} & \ldots & E_{m j} & \ldots & E_{m_{n} n_{m}}
\end{array}\right\}
\end{array}\right.
$$

Its important to note that generated ARR from BG should be considered with their component support because of it's graphical aspect:

$$
\begin{aligned}
C O M P(A R R) & \subset\{C, R, I, T F, G Y, D e, D f, S e, S f\} \\
= & \cup C O M P\left(A R R_{i}\right)
\end{aligned}
$$

Two component faults $F_{j}$ and $F_{i}$ are said strongly isolable (or discriminable) if and only if for given two over determined subsystems $M_{i}^{+}$and $M_{j}^{+}$, such that $: F_{j} \in M_{j}^{+}, F_{i} \in M_{i}^{+}$ and $\operatorname{COMP}\left(A R R_{j}\right) \cap C O M P\left(A R R_{i}\right)=\emptyset$,

$\operatorname{Card}(I s)$ can be used to evaluate the degree of isolability (strong, weak).

Let $\mathbf{r}$ the evaluation of ARRs: $r=\operatorname{Eval}(A R R)$, if $\mathbf{r}$ is near to zero, then the ARR is satisfied. If any fault on E component occurs, the ARR is violated then $\mathbf{r}$ generates an alarm (this is the detection step for which the condition of structural detectability is given above). The problem is how to isolate fault (to identify which component is faulty). In FDI model based is used the fault Boolean signature matrix (FSM). The Boolean value $s_{i j}$ equals 1 if the $i^{t h}$ residual is affected by the $j^{\text {th }}$ fault. The signature vector of each component fault $E_{j}$ is given by the row vector $V_{E j} j=1, \ldots, m=\left[\begin{array}{llll}s_{j 1}, & s_{j 2}, \ldots & s_{j n}\end{array}\right]$. A pair of component faults $\left(E_{j}, E_{i}\right)$ are isolable if their signature vector are different : $\forall \ell_{(\ell=1, \ldots, m)}, V_{E j} \neq V_{E \ell}(j \neq \ell)$

From the BG model as developed above, the FSM can be formed. Any element of the FSM matrix $E_{i j}$ represent BG elements covered by the causal paths during the unknown variable elimination procedure. 


\section{APPLICATION}

\section{A. Test Bench Description}

The test bench is used to test aircraft components to verify their functioning, their strength and endurance before integrating them in an airplane. Three configurations can be used, but in this study, we focus only on the two motors configuration given by Fig. 3. This configuration consists of positioning an industrial motor in front of an aeronautical motor. The actuator is a three-phase synchronous motor, couplings are used to make up the misalignment between the motor outlet and the inlet of the second motor. The first motor is controlled in torque while the second is controlled in angular velocity. The objective is to impose any torque trajectory (as disturbance) to the main motor. The connecting shaft (Fig.3) ensures the connection between the first and the second motor.

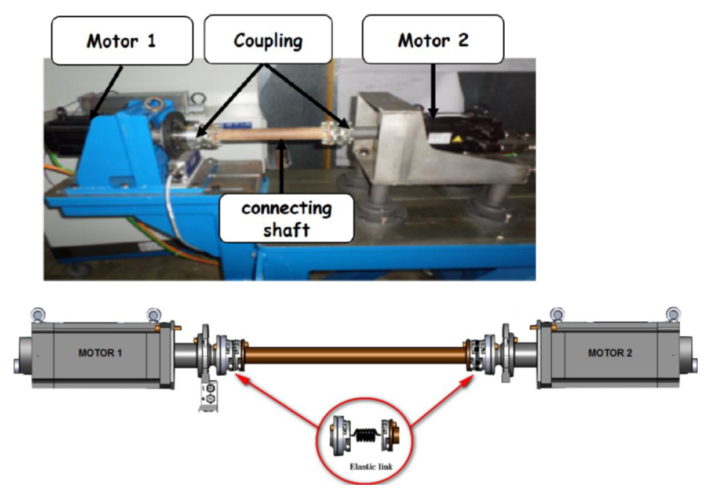

Fig. 3. Test bench overview and coupling shaft scheme

1) Word bond graph: The word bond graph (Fig.4) represents the technological level of the model. The global system is decomposed into different subsystems. In contrast to a conventional block diagram, the inputs and outputs in a word bond graph are not a signals but power variables. Two pairs of power variables are used: (torque-angular velocity $(\tau, \omega)$ for rotational mechanical energy and voltage-current $(V, i)$ for electrical energy.

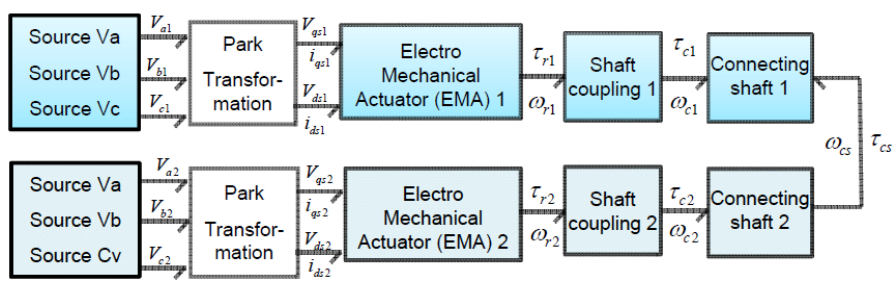

Fig. 4. Word Bond Graph of the global electromechanical test bench

\section{B. Bond Graph model of the induction motor}

The following assumptions are made for the development of a BG model for a synchronous machine: the stator windings are sinusoidally distributed along the air-gap; the stator slots cause no appreciable variation of the rotor inductance with rotor position; magnetic hysteresis is negligible; magnetic saturation effects are negligible.

In many cases, analysis of induction motors with space vector model is complicated due to the fact that we have to deal with variables of complex numbers. The most popular transform is between stationary $a-b-c$ frame quantities to synchronously rotating d-q quantities using well known Park's transformation (represented by the BG model of Fig. 5).

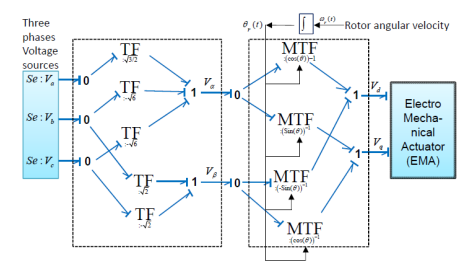

Fig. 5. BG model of Park's transformation

The global BG model of the induction motor is given Fig. 6. In a BG theory, the coupling between stator and rotor flux is modeled by multiport inertia I BG element. The constitutive equation is :

$$
\left[\begin{array}{c}
\frac{d \Phi_{d s}}{d t} \\
\frac{d \Phi_{r d}}{d t}
\end{array}\right]=\left[\begin{array}{cc}
L_{s} & L_{m} \\
L_{m} & L_{r}
\end{array}\right]\left[\begin{array}{c}
\frac{d i_{d s}}{d t} \\
\frac{d i_{r d}}{d t}
\end{array}\right]
$$

The modulated gyrator MGY represents the electromechanical power exchange. The system can be given under nonlinear state space equation $\dot{x}(t)=F(x(t), u)$ where $x$ is a state variable vector and $u$ the vector input represented by effort and flow sources in BG. From a BG model, the dimension of the state vector is equal to the number of dynamic BG element (I and C) in integral causality. The state variables are energy variables i.e. displacement for $\mathrm{C}$ elements and impulse for I element. For I element there are magnetic flux $\Phi=L i$ and angular momentum $M=J \omega$. The state vector does not appear on the Bond graph, but only its derivative. Thus, there are in the BG model $J \frac{d \omega}{d t}$ and $L \frac{d i}{d t}$. The state equations can be easily deduced from the BG or using specific softwares such as 20sim [10] or Symbols Shakti. [11]. The state equation is given below, where $I_{n}$ is unit triangular matrix and s Laplace derivative operator.

$$
\begin{aligned}
& \left\{\begin{array}{l}
L_{d} \frac{d i_{d s}}{d t}=-i_{d s} R_{s}+i_{q s} \omega_{s} L_{s}+i_{r q} \omega_{s} L_{m}-L_{m} s i_{r d} \\
L_{q} \frac{d q_{q s}}{d t}=-i_{q s} R_{s}-i_{d s} \omega_{s} L_{s}-i_{r d} \omega_{s} L_{m}-L_{m} s i_{r q} \\
L_{r} i_{r q}=-i_{r q} R_{r}+P i_{d s} L_{q} \omega_{r}-i_{r d} L_{m} \omega_{s}
\end{array}\right. \\
& \left\{L_{r} \frac{d i_{r q}}{d t}=-i_{r q} R_{r}+P i_{d s} L_{m} \omega_{r}-i_{r d} L_{m} \omega_{s}\right. \\
& -i_{d s} L_{m} \omega_{s}-L_{m} s i_{q s} \\
& L_{r} \frac{d i_{r d}}{d t}=-i_{r d} R_{r}-P i_{q s} L_{m} \omega_{r}+i_{r q} L_{m} \omega_{s}-L_{m} s i_{d s} \\
& J_{r} \frac{d \omega_{r}}{d t}=-\omega_{r} f_{r}=P L_{m}\left(i_{r d} i_{q s}-i_{r q} i_{d s}\right)-\omega_{r} f_{r} \\
& +\left[I_{n}\right]\left[\begin{array}{lllll}
V_{d} & V_{q} & V_{r q} & V_{r d} & \tau_{c s}
\end{array}\right]^{T}
\end{aligned}
$$

\section{Diagnosability analysis}

In our test bench, the used motor for driving the EMA is a permanent magnet synchronous motor with flux $\phi_{f}$. The 


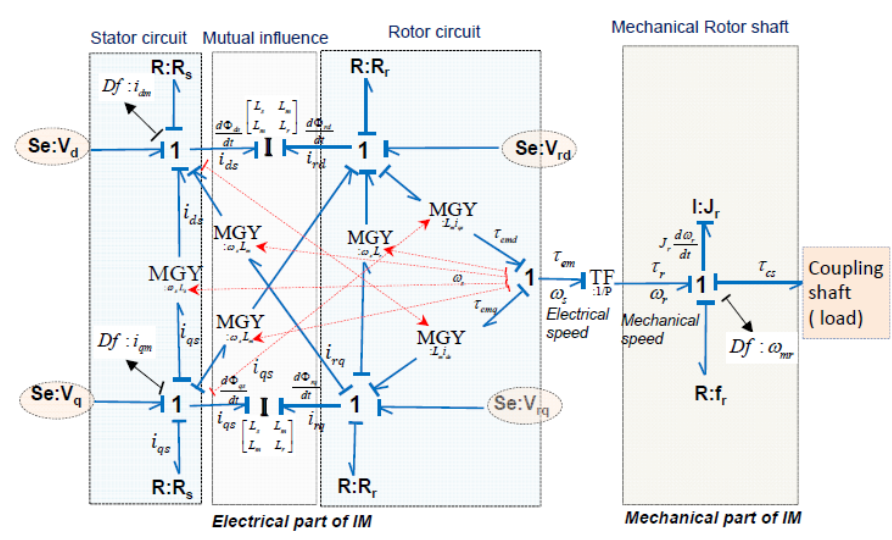

Fig. 6. Bond Graph model of the IM

global model in derivative causality suited for diagnosis is given Fig. 7. The BG model include electrical and mechanical dynamics of the two motors coupled by the coupling shaft angular modeled by angular momentum inertia and viscous friction represented by $I: J_{c s}$ and $R: R_{c s} \mathrm{BG}$ elements respectively. Coupling elasticities are represented by the two capacities $C: K_{c 1}$ and $C: K_{c 2}$ elements representing mechanical stiffness. $S S f$ and $S S e$ represent dualized flow (angular velocity, and current) and effort (torque) sensors. For simplicity all bonds are numbered, each number is associated with a pair of power variable (effort-flow).

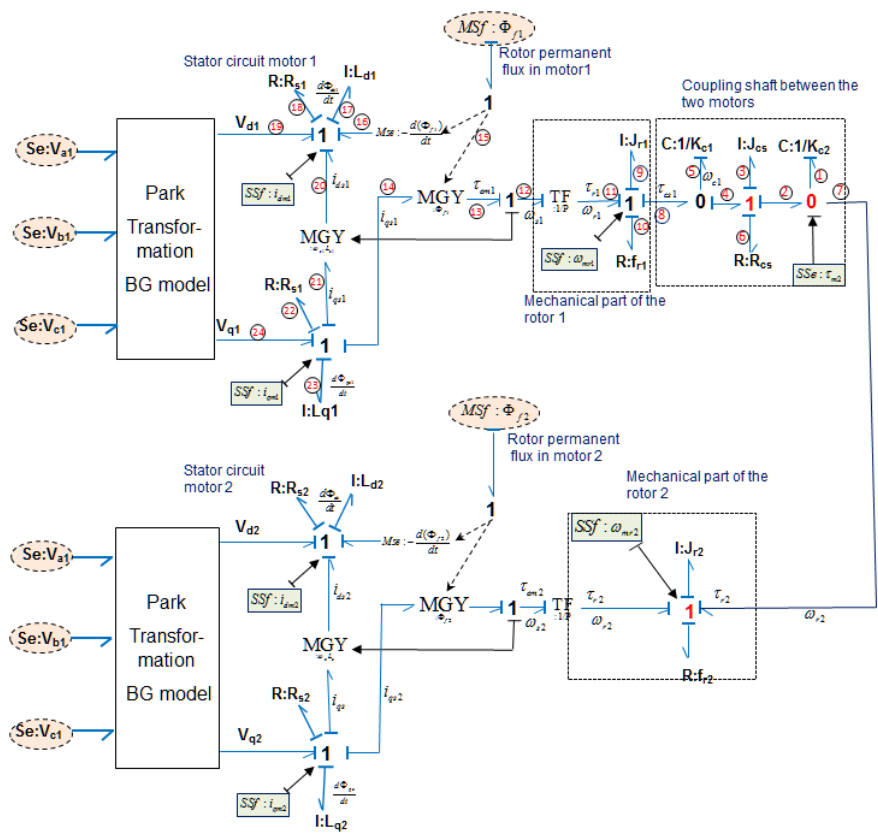

Fig. 7. Diagnosis BG of permanent synchronous motor

\section{ARR generation}

For simplicity and because of limited place, consider only the diagnosability of the motor 1 with the coupling shaft. The objective is to provide list of faults that can be detected and isolated using only given instrumentation architecture without any need of numerical calculation. For this task the structure of each ARR can be deduced from the bond graph model as developed in the paper. The model is given in derivative causality because in real systems initial conditions are not known. The ARR candidate are the constitutive equation of junctions associated with at least one sensor. Thus, are expected to deduce 4 ARRs. For pedagogical reason, consider in details the ARR generated from the junction "1" in the stator circuit motor 1 :

$$
\mathrm{J}_{1} \Rightarrow A R R_{1}=e_{19}-e_{18}+e_{20}-e_{17}+e_{16}=0
$$

$e_{19}, e_{18}, e_{20}, e_{17}$ and $e_{16}$ are unknown variables, they will be eliminated using covering causal (model inversion) from unknown to known ones (measured and sources). Measured variables are given by dualizing detectors (De and Df) into Signal Sources (SS) (SSf and SSe). The causal paths lead to oriented graphs showing how the unknown variables have been calculated (eliminated) as presented for this ARR by Fig. 8 .

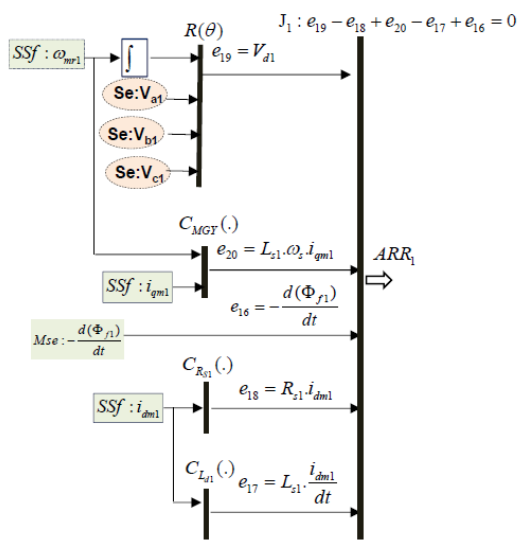

Fig. 8. Oriented graph leading to ARR generation

The analytical expression of the ARR is :

$$
\begin{aligned}
& A R R_{1}=\left[R_{\theta}\right]^{-1}\left(\omega_{m r 1}, V_{a 1}, V_{b 1}, V_{c 1}\right)-R_{s 1} \cdot i_{d m 1} \\
& +L_{s 1} \cdot \omega_{s} \cdot i_{q m 1}-L_{s 1} \cdot \frac{i_{d m 1}}{d t}+-\frac{d\left(\Phi_{f 1}\right)}{d t}
\end{aligned}
$$

where $\left[R_{\theta}\right]^{-1}$ is the inverse of Park's transformation. The components covered by the unknown variables elimination noted $C O M P\left(A R R_{1}\right.$ are :

$\operatorname{COMPS}\left(\mathrm{ARR}_{1}\right) \subset\left\{R_{s 1}, i_{d m 1}, \omega_{m r 1}, V_{a 1}, V_{b 1}, V_{c 1}, i_{q m 1}, L_{s 1}, \Phi_{f 1}\right\}$

It means that the faults which may affect this set of components can be detected by the fault indicator $A R R_{1}$. From the two junctions " 1 " in electrical and mechanical part, the following structural ARRs can be generated :

COMPS $\left(\mathrm{ARR}_{2}\right) \subset\left\{R_{s 1}, i_{d m 1}, \omega_{m r 1}, V_{a 1}, V_{b 1}, V_{c 1}, i_{q m 1}, L_{s 1}, \Phi_{f 1}\right\}$ $\operatorname{COMPS}\left(\mathrm{ARR}_{3}\right) \subset\left\{\Phi_{f 1}, i_{q m 1}, J_{r 1}, \omega_{m r 1}, f_{r 1}, J_{c s}, \tau_{m 2}, \omega_{m r 2}, k_{c 2}\right\}$

- Diagnosability Analysis

For diagnosability analysis a Fault Signature Matrix is built (Fig.9. $M_{a l 1}$ is a misalignment fault which may affect the rotor of motor 1. Only $A R R_{1}$ is sensitive to this fault while the candidate ARR is generated from the junction " 1 " representing conservative law dynamic equation of the rotor. $M_{b}$ and $I_{b}$ 
represent monitorability and Isolability indexes. All faults are detectable but no one is isolable (there is not a unique signature).

\begin{tabular}{|c|c|c|c|c|c|c|c|c|c|c|c|c|c|c|c|}
\hline Ib & 0 & 0 & 0 & 0 & 0 & 0 & 0 & 0 & 0 & 0 & 0 & 0 & 0 & 0 & 0 \\
\hline Mb & 1 & 1 & 1 & 1 & 1 & 1 & 1 & 1 & 1 & 1 & 1 & 1 & 1 & 1 & 1 \\
\hline $\begin{array}{c}\text { ARR/C } \\
\text { OMP }\end{array}$ & $R_{s 1}$ & $i_{d m 1}$ & $i_{q m 1}$ & $J_{r 1}$ & $f_{r 1}$ & $J_{c s}$ & $V_{a 1}$ & $V_{b 1}$ & $V_{c 1}$ & $L_{s 1}$ & $\Phi_{f 1}$ & $\omega_{m r 1}$ & $K_{c 2}$ & $\boldsymbol{\tau}_{m 2}$ & $M_{o l 1}$ \\
\hline ARR $_{1}$ & 1 & 1 & 1 & 0 & 0 & 0 & 1 & 1 & 1 & 1 & 1 & 1 & 0 & 0 & 0 \\
\hline ARR $_{2}$ & 1 & 1 & 1 & 0 & 0 & 0 & 1 & 1 & 1 & 1 & 1 & 1 & 0 & 0 & 0 \\
\hline ARR $_{3}$ & 0 & 0 & 1 & 1 & 1 & 1 & 0 & 0 & 0 & 0 & 1 & 1 & 1 & 1 & 1 \\
\hline
\end{tabular}

Fig. 9. Fault Signature Matrix for Diagnosability analysis

\section{- Sensor placement and causal loop}

As noted in II-C the system is not observable if the over constrained and just-constrained subsystems is not causal i.e. there is a differential loop. Another ARR should have been generated from "0" junction linked to the torque sensor $S S e$ : $\tau_{m 2}$, but one of the covering causal paths to eliminate unknown variables presents a differential causal loop. Precisely, it is the causal path to eliminate $f_{2}$ as shown by dashed line and corresponding path in Fig. 10. This causal could be "broken" adding new sensors, for instance an angular velocity sensor for the coupling shaft.
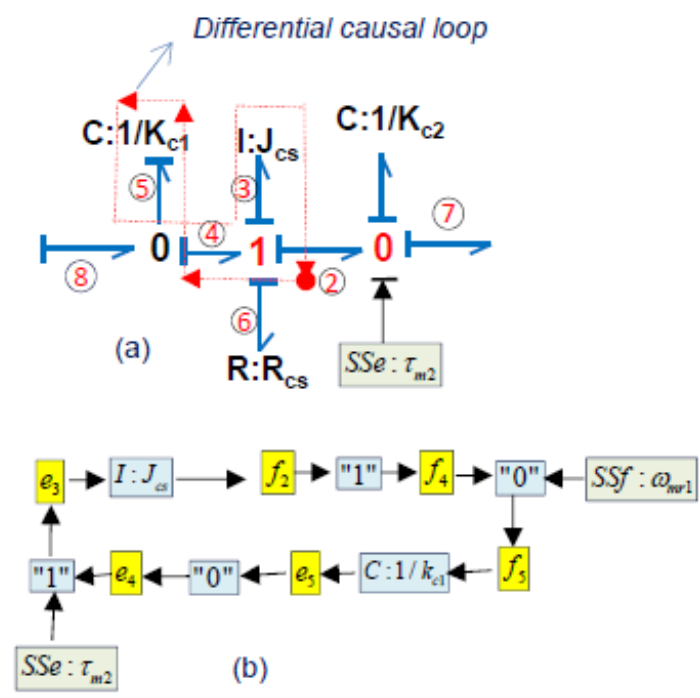

Fig. 10. No diagnosable subsystem because of differential causal loop

Finally the diagnosability performances are low, specially concerning the motor jamming represented in the model by the friction coefficient $f_{r 1}$. This fault is weakly isolable while the signature vector of this parameter [001] has seven identical signatures.To improve isolability performances a sensor placement should be proposed or to design an observers (software sensor) to estimate some variables.

\begin{tabular}{|c|c|}
\hline $\mathrm{i}_{d s}, i_{q s}$ & Stator currents for transformed induction motor model [A] \\
\hline $\mathrm{L}_{m}$ & Stator and rotor mutual inductance [H] \\
\hline $\mathrm{L}_{d}, L_{q}$ & Rotor self inductance in d-q refernce [H] \\
\hline $\mathrm{L}_{s}, L_{r}$ & Rotor and stator self inductance $[\mathrm{H}]$ \\
\hline $\mathrm{R}_{s}$ & Stator winding resistance $[\Omega]$ \\
\hline $\mathrm{T}_{e}$ & Electromechanical torque $[\mathrm{Nm}]$ \\
\hline$\omega_{r}$ & Rotating speed of shaft [rad/s] \\
\hline$\omega_{s}$ & Synchronous speed [rad/s] \\
\hline$\Phi_{d s}, \Phi_{q s}$ & Stator flux linkages for transformed induction motor model [Wb-t] \\
\hline$\Phi_{f}$ & Rotor flux linkages [Wb-t] \\
\hline $\mathrm{P}$ & Number of pole pairs \\
\hline \multicolumn{2}{|c|}{ TABLE I } \\
\end{tabular}

\section{Conclusion}

The paper concerns the first part of the project, and deals with use of bond graph model as unified and multidisciplinary tool not only for modeling but also for structural diagnosability analysis (which faults which may affect component including sensors can be detected an isolated ?) and sensor placement proposition to make the system diagnosable. An application to the induction motor as main component in an EMA is used for illustration. The main fault to be monitored according the industrial is the Jamming of the motor. Based on existing instrumentation architecture, the signature vector of this fault is similar to six others signatures. Future work consists in sensor placement proposition to satisfy specifications and real time validation of the diagnosis algorithms.

\section{ACKNOWLEDGMENT}

The authors would like to thank CERTIA company for their financial support.

\section{REFERENCES}

[1] R. Isermann, "Process fault detection based on modelling and estimation methods : A survey," Automatica, vol. 20, pp. 387-404, 1994.

[2] H. Nejjari and M. Benbouzid, "Monitoring and diagnosis of induction motors electrical faults using a current parks vector pattern learning approach," IEEE Transactions on Industry Applications, vol. 36, 2000.

[3] B. ould Bouamama, G. Biswas, R. Loureiro, and R. Merzouki, "Graphical methods for diagnosis of dynamic systems: Review," Annual Review in Control, vol. 38, Issue 2, pp. 199-219, 2014.

[4] B. Ould-Bouamama, K. Medjaher, M. Bayart, A. Samantaray, and B. Conrard, "FDI of smart actuators using bond graphs and external models," Control Engineering Practice (CEP), vol. 13, no. 2, pp. 159175, 2004.

[5] H. Paynter, Analysis and design of Engineering Systems. M.I.T. Press, 1961.

[6] M. R. Maurya, R. Rengaswamy, and V.Venkatasubramanian, "A signed directed graph and qualitative trend analysis-based framework for incipient fault diagnosis," Chemical Engineering Research and Design, vol. 85, no. 10, pp. 1407-1422, 2007.

[7] M. Blanke, M. Kinnaert, J. Lunze, and M. Staroswiecki, Diagnosis and fault-tolerant control. Springer Verlag, 2010.

[8] B. Ould-Bouamama, A. K. Samantaray, M. Staroswiecki, and G. Dauphin-Tanguy, "Derivation of constraint relations from bond graph models for fault detection and isolation," in Int. Conf. Bond Graph Modeling and Simulation, 2003, pp. 104-109.

[9] B. Ould-Bouamama, M. Staroswiecki, and A. Samantaray, "Software for supervision system design in process engineering industry," in 6th IFAC IFAC SAFEPROCESS. IFAC, september 2006, pp. 691-695.

[10] C. Products, "20-sim, the software for modeling dynamic systems," http://www.20sim.com/, 20109.

[11] A. Mukherjee and R. Karmkar, Modelling and Simulation of Engineering Systems Through Bond Graph. Norosa Publishing Hous, 2000. 